\title{
Response to influenza immunisation in asymptomatic HIV infected men
}

\author{
Mia Huengsberg, Mina P Chakraverty, Gill Cooper, Mohsen Shahmanesh
}

\begin{abstract}
Objective-This study aimed to determine if patients with HIV infection can develop a significant antibody response to influenza immunisation, and whether such immunisation is detrimental to the progression of their HIV illness.

Design-Prospective, non-randomised study.

Methods-The titres of antibody response to influenza immunisation were determined in 44 asymptomatic HIV positive men and compared with 16 HIV presumed seronegative controls. The magnitude of response were correlated with patients' CD4 lymphocyte counts. The rate of CD4 lymphocyte count decline over a median of 12 months before and after immunisation were also evaluated. Results-Thirty-two of the 44 HIV positive subjects $(73 \%)$ were able to mount a four-fold or greater response to at least one of the influenza strains, and 14 of them $(32 \%)$ did so to all four strains. In comparison, 15 of the 16 controls $(93.5 \%)$ had a four-fold or greater response to at least one, and six of them (38\%) to all influenza strains, which was not significantly different from the HIV positive group. The magnitude of increase in antibody titre was not significantly different between the two groups either. CD4 lymphocyte count change for a median of 12 months after immunisation was not different from a median of 12 months before immunisation.
\end{abstract}

Conclusions-Asymptomatic HIV infected patients are able to mount antibody response to influenza immunisation, which appears to be safe in respect to HIV illness progression in the short term.

\section{(Genitourin Med 1995;71:355-357)}

Keywords: influenza; immunisation; HIV

\section{Introduction}

The influenza virus can have devastating effect on public health: in each of the 19 epidemics that occurred in the United States between 1957 and 1986, influenza accounted for more than 10000 excess deaths. ${ }^{1}$ Influenza vaccine is $75 \%$ effective in preventing complications and death in susceptible individuals. ${ }^{2}$ Its usage in persons affected by human immunodefi- ciency virus (HIV) has been recommended by public health advisory bodies of the United States, ${ }^{3}$ some European countries ${ }^{4}$ and the United Kingdom. ${ }^{5}$

Our study aimed to determine if patients with HIV infection develop a significant antibody response to influenza vaccination, whether the antibody response is dependent on T-helper lymphocyte (CD4) count and whether the vaccine adversely affects the patients' immunity by altering the rate of CD4 decline.

\section{Materials and methods}

Study population

Fifty-six asymptomatic (CDC Stage II/III) ${ }^{6}$ HIV infected men attending the Genitourinary (GU) Medicine Clinic of Birmingham General Hospital and Birmingham Heartlands Hospital for outpatient follow-up were invited to participate in the study. Forty-four patients were recruited and completed the study. Their median age was 32 years (range 17 to 54 ); 43 were homosexuals, and one was an intravenous drug user. Their median $\mathrm{CD} 4$ count immediately before vaccination was $330 \times$ $10^{6} / \mathrm{ml}$ (range 130-1320 $\times 10^{6} / \mathrm{ml}$ ). Seventeen of them were taking antiretroviral drugs at the time of the study.

Patients were divided into three groups according to their CD4 lymphocyte count at the beginning of the study: CD $4<200 \times$ $10^{6} / \mathrm{ml}(\mathrm{N}=6), \quad \mathrm{CD} 4=200-500 \times 10^{6} / \mathrm{ml}$ $(\mathrm{N}=23)$ and $\mathrm{CD} 4>501 \times 10^{6} / \mathrm{ml}(\mathrm{N}=15)$.

Sixteen male members of staff (including doctors and nurses working in our department and medical students doing GU medicine attachment at the time of the study) were recruited as controls. Their median age was 28 years (range 20 to 51). Fifteen had no known risk factors for HIV infection; one was homosexual who had never practised unsafe sex. HIV test was not performed in this group, as the chance of seropositivity was considered extremely small. None had any known medical illnesses or took long-term medications. Informed consent was obtained from all patients and controls. Side effects were monitored by self-reporting, regular physical examination as well as regular full blood counts, liver and renal function tests were routinely performed.

\section{Vaccination and laboratory investigation}

The study was performed between October 1992 and January 1993. All patients and con- 
trols were vaccinated with one single intramuscular injection of Influvac (by Duphar) containing $15 \mu \mathrm{g}$ of each of the influenza viral strains A/Singapore/6/86 H1N1, A/Beijing/ $353 / 89 \mathrm{H} 3 \mathrm{~N} 2$ and $\mathrm{B} /$ Yamagata/16/88. The vaccine was manufactured for the winter of $1992 / 93$. None of the subjects had a history of possible influenza during that season prior to vaccination.

Venous blood was obtained within 4 hours prior to, and 15 to 110 days (median 31.5 days) after Influvac in the patient group, and 27 to 44 days (median 31 days) after Influvac in the control group. Approximately $1 \mathrm{ml}$ of serum was stored at $-20^{\circ} \mathrm{C}$ and transported to PHLS Laboratory in Colindale, London. Antibody levels to each of the above influenza virus strains including $B / P a n a m a / 45 / 90$ which was the alternative influenza $B$ component recommended by the WHO for the 1992/93 influenza vaccine, were assayed in batches by haemagglutination inhibition against 8AD antigen. A four fold rise in antibody titre or a level of 1:40 was considered an adequate response. $^{7}$

Serial CD4 lymphocyte count were obtained according to usual clinical routine in all patients at 3 monthly intervals. All available CD4 lymphocyte count for a median of 12 months before (range 1-17) and 12 months (range 5-17) after the vaccination were collected in all subjects. The rate of CD4 decline was calculated by averaging the mean monthly decline between each separate measured CD4 count.

\section{Statistical analysis}

Analysis was performed with median values and fold increase (calculated as the ratio of postvaccination to prevaccination titre) of each of the antibody titres before and after

Table 1 Median fold increase in influenza antibody titre after vaccination

\begin{tabular}{lllll}
\hline & $\begin{array}{lll}C D 4 \\
N=6\end{array}$ & $\begin{array}{l}C D 4200-500 \\
N=23\end{array}$ & $\begin{array}{l}C D 4<500 \\
N=15\end{array}$ & $\begin{array}{l}\text { Normal } \\
N=16\end{array}$ \\
\hline Singapore & $2 \cdot 0$ & $2 \cdot 0$ & $4 \cdot 0$ & $4 \cdot 0$ \\
& $(2 \cdot 0-3 \cdot 3)$ & $(2 \cdot 0-3 \cdot 1)$ & $(2 \cdot 75-13 \cdot 0)$ & $(2 \cdot 0-9 \cdot 0)$ \\
Beijing & $4 \cdot 0$ & $4 \cdot 0$ & $2 \cdot 0 \star$ & $16 \cdot 0$ \\
& $(1 \cdot 0-23 \cdot 4)$ & $(2 \cdot 0-12 \cdot 4)$ & $(2 \cdot 0-6 \cdot 0)$ & $(4 \cdot 0-32 \cdot 0)$ \\
Yamagata & $3 \cdot 0$ & $4 \cdot 0$ & $4 \cdot 0$ & $12 \cdot 0$ \\
& $(1 \cdot 4-11 \cdot 7)$ & $(2 \cdot 0-12 \cdot 0)$ & $(2 \cdot 0-8 \cdot 0)$ & $(1 \cdot 0-19 \cdot 8)$ \\
Panama & $3 \cdot 0$ & $4 \cdot 0$ & $4 \cdot 0$ & $8 \cdot 0$ \\
& $(2 \cdot 0-6 \cdot 6)$ & $(2 \cdot 0-8 \cdot 0)$ & $(2 \cdot 0-8 \cdot 0)$ & $(2 \cdot 0-16)$ \\
\hline
\end{tabular}

Figures in brackets show $95 \%$ confidence interval (1-Sample Sign Confidence Interval); asterisk indicates result significantly different from control group (Mann-Whitney $U$ Test: $W=163 \cdot 5$; $\mathrm{p}=0.004)$.

Table 2 Median antibody titre achieved after influenza vaccination

\begin{tabular}{lllll}
\hline & $C D 4<200$ & $C D 4200-500$ & $C D 4<500$ & $\begin{array}{l}\text { Normal } \\
N=16\end{array}$ \\
& $N=6$ & $N=23$ & $N=15$ & \\
\hline Singapore & $80 \cdot 0$ & $160 \cdot 0$ & $160 \cdot 0$ & $160 \cdot 0$ \\
& $(80 \cdot 0-468 \cdot 6)$ & $(80 \cdot 0-160 \cdot 0)$ & $(80 \cdot 0-260 \cdot 2)$ & $(160 \cdot 0-320 \cdot 0)$ \\
Beijing & $20 \cdot 0 \star$ & $40 \cdot 0$ & $40 \cdot 0 \dagger$ & $120 \cdot 0$ \\
& $(10 \cdot 0-160 \cdot 0)$ & $(20 \cdot 0-160 \cdot 0)$ & $(20 \cdot 0-80 \cdot 0)$ & $(70 \cdot 5-640)$ \\
Yamagata & $40 \cdot 0$ & $80 \cdot 0$ & $40 \cdot 0$ & $160 \cdot 0$ \\
& $(20 \cdot 0-117 \cdot 0)$ & $(40 \cdot 0-160 \cdot 0)$ & $(20 \cdot 0-130 \cdot 1)$ & $(40 \cdot 0-320 \cdot 0)$ \\
Panama & $40 \cdot 0$ & $80 \cdot 0$ & $40 \cdot 0$ & $80 \cdot 0$ \\
& $(20 \cdot 0-80 \cdot 0)$ & $(40 \cdot 0-124 \cdot 2)$ & $(20 \cdot 0-80 \cdot 0)$ & $(20 \cdot 0-160 \cdot 0)$ \\
\hline
\end{tabular}

Figures in brackets show $95 \%$ confidence interval (1-Sample Sign Confidence Interval); asterisk indicates result significantly different from control group (Mann-Whitney $U$ Test: ${ }^{\star} W=41 \cdot 0$; $\mathrm{p}=0.04 ; \mathrm{W}=171 \cdot 0, \mathrm{p}=0.007)$. vaccination. Comparison was made between HIV positive patients and staff controls, as well as between patients of different CD4 lymphocyte subgroups. MINITAB statistical software package was utilised; Mann-Whitney U test was performed when comparing median values and chi-square test for proportions.

\section{Results}

Thirty-two of the $44 \mathrm{HIV}$ positive subjects (73\%) were able to mount a four-fold or more response to at least one of the virus strains, and 14 of them $(32 \%)$ did so to all four strains. In comparison, 15 of the 16 controls (93.5\%) had a four-fold or more response to at least one, and six (25\%) of them to all strains. Twenty two $(50 \%)$ of the patients and $10(63 \%)$ of controls achieved a titre of $1: 40$ to all four strains of antigens after immunisation, a level considered protective against influenza. ${ }^{7}$ Though showing a slight trend towards better response in the control group, the differences were not statistically significant.

When the median fold-increase of titre of each patient subgroup (according to their CD4 lymphocyte count) was compared with that of the control group, there was no significant difference in all but one antibody titre rise between each group and normal controls (table 1). The median post vaccination titre to the four influenza antigens in each patient subgroup were lower than the corresponding titre in the control group (table 2), but with the exception of titre to A/Beijing/353/89 H3N2 strain, the trend did not reach statistical significance.

The median CD4 lymphocyte count for the patients was $330 \times 10 \%$ ml $\quad(130-1320$ $\times 10^{6} / \mathrm{ml}$ ) immediately before and $350 \times$ $\left.10 \% / \mathrm{ml} 10-950 \times 10^{6} / \mathrm{ml}\right) 3$ months post vaccination. CD4 lymphocyte counts before (median of 3, range 1-9 samples) and after (median of 4, range 1-11 samples) immunisation were analysed. The median rate of CD4 lymphocyte decline was calculated as $1.3 \times$ $10 \% / \mathrm{ml}$ (mean -2.97 ; SD $28 \cdot 5$; SE $4 \cdot 1$; range -123 to 65 ) per month prior to Influvac injection, and $2.65 \times 10^{6} / \mathrm{ml}$ (mean 6.61 ; SD $21 \cdot 1$; SE $3 \cdot 1$; range -33 to 75 ) per month after the injection. The difference was not significant (Mann-Whitney $U$ test: $W=2023 \cdot 0, \quad p=$ $0 \cdot 11$ ). Analysis of the square roots of mean CD4 lymphocyte decline ${ }^{8}$ also failed to show any difference before and after immunisation.

Influvac was well tolerated by patients and controls without major side effects. No patients died within 6 months of receiving the vaccination.

\section{Discussion}

This study demonstrates that asymptomatic HIV positive patients are able to mount adequate antibody response to influenza vaccine. Seventy-three percent of HIV positive patients were able to mount a four-fold or more antibody response to at least one of the four influenza antigens, and $50 \%$ achieve protec- 
tive antibody response to all vaccinated strains.

When compared with immunocompetent controls, HIV positive patients seem to mount a lower antibody response after vaccination. However, the difference was not statistically significant in all but one strain. We were unable to detect even a trend towards higher antibody response in patients with good CD4 count compared with those with low CD4 count. This is in contrast to the study by Kroon et $a l^{10}$ who reported that antibody response in HIV patients seemed to correlate with their CD4 count. However, unlike Kroon's study, our patient group did not include those with CD4 lymphocyte count less than $100 \times 10^{6} / \mathrm{ml}$.

Even though the timing of testing for antibody response after vaccination varied between individuals (range 15-110 days), we do not feel that this has significantly influenced our results. Influvac antibody response is expected to occur within 2 weeks after vaccination and remain stable for at least six months.

This study did not address the question of clinical efficacy of the vaccine. Cost-effective analysis $^{11}$ has shown that routine recommendation of influenza immunisation to HIV infected patients is a less efficient policy compared with pneumococcal vaccination, as the case fatality of influenza is lower, immunodeficiency only minimally increases the risk of contracting influenza, and vaccination is necessary annually. However, influenza can predispose to bacterial pneumonia in immunocompetent adults; in immunocompromised patients, symptoms may be prolonged and risk of complications increased. ${ }^{12}$ In the United States, up to a third of HIV infected adolescents and adults receive influenza vaccination. ${ }^{13}$ This study, together with others, have shown that if influenza immunisation is considered appropriate is a particular individual, adequate antibody response can be expected in asymptomatic patients, especially if the CD4 lymphocyte count is $>100 \times 10^{6} / \mathrm{ml} .^{9}$

Our study confirms the safety of the influenza vaccine. We were unable to demonstrate any deleterious effect of the vaccine on the course of HIV infection as evidenced by our observation on CD4 lymphocyte count. ${ }^{14}$ Three months after immunisation, there was no change in the mean CD4 lymphocyte count. A median of 12 months later, the rate of CD4 lymphocyte decline was not different from that prior to immunisation. This confirms findings of other studies ${ }^{910}$ which showed no unexpected change in CD4 lymphocyte count after influenza immunisation.

We thank Dr E Boxall, Dr S Skidmore and Mrs Julie King of Regional Virus Laboratory, Birmingham Heartlands Hospital for their assistance in handling and transporting the serum samples.

This study was not formally considered by the University of Birmingham Research Ethics Committee. However, the project was discussed with the Chairman, who gave his verbal approval of the study.

1 Immunisation Practices Advisory Committee (ACIP): Prevention and control of influenza: Part I, vaccines. MMWR 1989;38:297-8, 303-11.

2 Patriarca PA, Weber JA, Parker RA et al: Efficacy of Patriarca PA, Weber JA, Parker RA et al: Efficacy of
influenza vaccine in nursing homes: reduction in illness and complications during influenza A (H3N2) epidemic. fAMA 1985;253:1136-9

3 Immunisation Practices Advisory Committee: Prevention and control of influenza. MMWR 1993;42:1-14.

4 Health Council of the Netherlands: Committee on influenza vaccination. Influenza Vaccination: Season 1993-1994. The Hague: Health Council of The Netherlands; 1993 (1993/16).

5 Joint Committee on Vaccination and Immunisation: Influenza. In Immunisation against Infectious Diseases. London: HMSO; 1992:95-9.

6 Centres for Disease Control: Revision of the CDC surveillance case-definition for acquired immunodeficiency syndrome. $M M W R$ 1987;36(suppl):1S-15S

7 Pereira MS, Chakraverty P, Schild GC, Coleman MI, Doudle WR. Prevalence of antibody to current influenza viruses and effect of vaccination on antibody response. $B M \mathcal{F}$ 1972;4:901-3.

8 Boutitie F, Pocock SJ: Predictive value of repeated measurements of CD4 lymphocyte counts on progression to AIDS. AIDS 1994;8:35-41.

9 Huang KL, Ruben FL, Rinaldo CR, Kingsley L, Lyter DW, Ho M: Antibody response after influenza and pneumococcal immunisation in HIV-infected homosexual mococcal immunisation in HIV

10 Kroon FP, van Dissel JT, de Jong JC, van Furth $\mathrm{R}$ : Antibody response to influenza, tetanus and pneumococcal vaccines in HIV-seropositive individuals in relation to the number of CD4 + lymphocytes. AIDS 1994;8: 469-76.

11 Rose DN, Schechter CB, Sacks HS: Influenza and pneumococcal vaccination for HIV-infected patients: a policy analysis. Am F Med 1993;94:160-8.

12 Safrin S, Rush J, Mills J: Influenza in patients with human immunodeficiency infection. Chest 1990;98:33-7.

13 Wortley PM, Farizo KM and the adult and adolescent spectrum of HIV disease project group: pneumococcal and influenza vaccination levels among HIV-infected ad influenza vaccination levels among HIV-infected adolescents and adults receiving mec
United States. AIDS 1994;8:941-4.

14 De Gruttola V, Wulfsohn M, Fischl MA, Tsiatis A: Modeling the relationship between survival and CD4 lymphocytes in patients with AIDS and AIDS-related complex. $\mathcal{F}$ Acquir Immune Defic Syndr 1993;6:359-65. 